\title{
Compatibility of QCD sum-rules and Hadron field theory in a dense medium
}

\author{
R. Aguirre \\ Departamento de Física, Fac. de Ciencias Exactas, \\ Universidad Nacional de La Plata. \\ C. C. 67 (1900) La Plata, Argentina.
}

\begin{abstract}
The compatibility of the QCD sum rules and effective hadronic models predictions are examined. For this purpose we have considered the results for the nucleon selfenergy in a dense hadronic environment provided by two independent QCD sumrules calculations. They are immersed in a theory of hadronic fields giving rise to non-linear interactions, whose vertices are parameterized in different ways. Although all of them reproduce the self-energy used as input, very different descriptions of nuclear observables are obtained. Only under very definite circumstances we have found an acceptable agreement with the nuclear matter properties. To achieve this, phenomenological parameters are not required at all.
\end{abstract}

Key words: Relativistic hadronic models, QCD sum rules

PACS: 24.85.+p, 21.30.Fe, 12.38.Lg, 21.65.+f

Currently, there is a noticeable interest in interpreting and formalizing the low energy manifestations of the fundamental theory of the strong interactions. However, the mathematical complexity that Quantum Chromodynamics (QCD) exhibits in this regime makes impossible to reach this aim. Several approaches were proposed to circumvent this difficulty, such as lattice simulations, QCD sum-rules, and effective models, for instance Nambu- Jona Lasinio, bag-like models, and chiral perturbation theory. Each of these treatments emphasizes some aspects of QCD, considered as the most relevant in the hadronic phase. Contact with low energy physics is ensured by fixing some model parameters, such as hadronic masses and decay constants to its experimental values. It is generally believed that for high density and/or temperatures, the symmetries of the fundamental theory would be recovered, therefore effective models should reproduce this feature to some degree.

The low to medium density states of nuclear matter have been extensively studied by using nuclear potentials. Most of the nuclear structures, reactions, and dispersion phenomenology have been successfully described in this way. However, these formulations lack 
of Lorentz covariance and they violate causality when extrapolated to higher densities. The requirements of covariance and causality can be fulfilled properly within the hadronic field theory (HFT), whose prototype the Quantum Hadrodynamics [1] usually takes dense nuclear matter as its reference state. This practice contrasts with certain nuclear potential treatments that refer to in- vacuum scattering lengths, ascribing this feature to a more fundamental instance of nuclear potentials as compared with HFT. Inspired in this viewpoint, several investigations have been made in order to make compatible both schemes, due to the simplicity and accuracy of the HFT treatment. The in-medium self-energy of the nucleon plays a key role in these attempts, as it is evaluated in both approaches and then equated to extract medium dependent vertices $[2,3,4,5]$.

The idea behind this procedure is that when a piece of information provided by another field of research is coherently inserted into the framework of the HFT, a simpler and more intuitive description is obtained instead of stating first principles interactions and performing involved calculations. This procedure is specially suited for high density systems since the details of the interactions are faded out in favor of a statistical average. A significative exemplification is given by the Brown-Rho scaling of hadronic masses. Taking into account the chiral and scale symmetries of QCD solely, an approximate scaling law for the in-medium hadronic masses was derived in [6]. This hypothesis was applied to describe heavy ion collision, reaching an excellent agreement with the experimental results for the low mass dilepton production rate [7].

On the contrary, going from low to higher energies, references $[2,3,4,5]$ take nuclear potentials as the starting point and results are extrapolated to the medium-high dense nuclear matter and atomic nuclei. In ref. [4] it was stressed that a coherent model requires that the medium dependence of the above mentioned vertices must be introduced in terms of hadronic fields. Otherwise, the rearrangement self-energies are missed and thermodynamical consistency is not satisfied.

In this work, we try to match QCD inspired results with the HFT formalism, by introducing field dependent strength couplings as proposed in [4]. For this purpose, we have chosen as inputs the nucleon self-energies evaluated within the QCD sum-rules approach $[8,9,10,11,12]$. We have compared different field parameterizations of the vertices, and examined the ability of our results to fit the nuclear matter phenomenology.

QCD sum-rules is a powerful procedure to reveal the QCD foundations of certain hadronic properties. This method relies on the evaluation of correlation functions in terms of quarks and gluons degrees, then applying the operator product expansion it is possible to express them as combinations of perturbative contributions and condensates (non-perturbative). In the last step, results are connected with the hadronic counterpart, from which information on the spectral function can be inferred. The method was developed to study meson [8] as well as baryon [9] properties in vacuum, it was subsequently generalized to study finite density systems $[10,11,12]$. We have used the in-medium nucleon self-energies provided by references $[11,12]$, more precisely the simplified expressions given there as functions of the baryonic density. Both investigations include non-perturbative contributions representing one meson exchange, the structure of the nucleon, and multiple meson exchange. Nevertheless, they differ in the criteria used to simplify the final formulae. The results of ref. [12] keep all the contributions averaged over the Borel mass parameter; whereas, in [11] only the leading term in the operator product expansion is retained, and 
the Borel mass is fixed in order that the effective nucleon mass goes to its experimental value in vacuum. In the latter case, a linear dependence on the density is obtained for both the scalar and vector nucleon self-energy.

As it was above mentioned, the Density Dependent Hadron Field Theory (DDHFT) [4,5] attempts to insert the nucleon self-energy supplied by Dirac-Brueckner calculations with one boson exchange potentials, into a hadronic field description of the nuclear matter phenomenology. In general terms, we have adopted the procedure described in ref. [4] to merge dynamically the external input into a field model, and we will indicate where we depart from it.

We start with a lagrangian for nucleons $(\psi)$ and scalar and vector mesons $(\sigma$ and $\omega$ respectively), resembling the QHD-I model of Walecka [1] for isospin symmetric nuclear matter,

$$
\mathcal{L}=\bar{\psi}\left(i \not \partial-M+G_{s} \sigma-G_{v} \not\right) \psi+\frac{1}{2}\left(\partial_{\mu} \sigma \partial^{\mu} \sigma-m_{s}^{2} \sigma^{2}\right)-\frac{1}{4} F_{\mu \nu} F^{\mu \nu}+\frac{1}{2} m_{v}^{2} \omega_{\mu} \omega^{\mu}
$$

where $F_{\mu \nu}=\partial_{\mu} \omega_{\nu}-\partial_{\nu} \omega_{\mu}$ has been used, and the meson masses have been fixed at the empirical values $m_{v}=783 \mathrm{MeV}, m_{s}=550 \mathrm{MeV}$. The main difference is that $G_{s}$ and $G_{v}$ are not coupling constants as in the Walecka model, but they are field functionals. Since these vertices are Lorentz scalars, we expect that the dependence on the fermion fields appears through the bilinear combinations $s_{1}(x)=\bar{\psi}(x) \psi(x), s_{2}(x)=\sqrt{n_{\mu}(x) n^{\mu}(x)}$, with $n_{\mu}(x)=\bar{\psi}(x) \gamma_{\mu} \psi(x)$ the baryonic current. Ref. [4] only developed the dependence on $s_{1}$ or $s_{2}$; in this paper, we also consider the eventual dependence on the meson fields but neglect derivatives of any of the fields, namely $G_{s, v}=G_{s, v}\left(s_{1}, s_{2}, s_{3}, s_{4}\right)$, with $s_{3}=\sqrt{\omega_{\mu} \omega^{\mu}}$, and $s_{4}=\sigma$. The field equations are obtained in the usual way, by minimizing the action

$$
\begin{gathered}
\left(i \not \partial-M+G_{s} \sigma-G_{v} \phi+\bar{\psi} \sum_{1}^{2} \frac{\partial G_{s}}{\partial s_{k}} \frac{\partial s_{k}}{\partial \bar{\psi}} \sigma-\bar{\psi} \sum_{1}^{2} \frac{\partial G_{v}}{\partial s_{k}} \frac{\partial s_{k}}{\partial \bar{\psi}} \psi\right) \psi=0 \\
\left(\square+m_{s}^{2}\right) \sigma=\bar{\psi}\left(G_{s}+\frac{\partial G_{s}}{\partial s_{4}} \sigma-\frac{\partial G_{v}}{\partial s_{4}} \psi\right) \psi \\
\partial_{\mu} F^{\mu \nu}+m_{v}^{2} \omega^{\nu}=\bar{\psi}\left[G_{v} \gamma^{\nu}+\frac{d s_{3}}{d \omega_{\nu}}\left(-\frac{\partial G_{s}}{\partial s_{3}} \sigma+\frac{\partial G_{v}}{\partial s_{3}} \psi\right)\right] \psi
\end{gathered}
$$

The two last terms within brackets in Eq. (2) evaluated in the mean field approximation can be regarded as the scalar and vector components of the nucleon self-energies. In order to make the problem solvable, only a set of variables $s_{k}$ can be taken into account in the evaluation of the functionals $G_{s}$ and $G_{v}$. We have considered the following cases: a) both vertices depend only on $s_{2}$, b) $G_{s}$ depends on $s_{1}, G_{v}$ depends on $s_{2}$, and c) $G_{s}$ depends on $\sigma, G_{v}$ depends on $s_{3}$. The case (a) has been developed in great detail in ref. [4], the same as case (b) they produce meson equations resembling those of the Walecka model, and nucleon self-energies with the rearrangement contributions added. In the instance (c) the self-energies have the same structure as in QHD-I, but the source term of the meson 
equations are modified.

The mean field approximation (MFA) is suited to describe static homogeneous nuclear matter. Within this scheme, meson fields are replaced by their uniform mean values and bilinear combinations of spinors are replaced by their expectation values. Therefore within MFA, Eqs. (2-4) reduce to

$$
\begin{aligned}
0 & =\left(i \not \partial-M+\Sigma_{s}-\not\right) \psi \\
m_{s}^{2} \tilde{\sigma} & =<\bar{\psi} G_{s} \psi>+<\bar{\psi} \frac{\partial G_{s}}{\partial \sigma} \psi \sigma>-<\bar{\psi} \frac{\partial G_{v}}{\partial \sigma} \psi \phi> \\
m_{v}^{2} \tilde{\omega}^{\nu} & =<\bar{\psi} G_{v} \gamma^{\nu} \psi>-<\bar{\psi} \frac{\partial G_{s}}{\partial s_{3}} \frac{\omega^{\nu}}{s_{3}} \psi \sigma>+<\bar{\psi} \frac{\partial G_{v}}{\partial s_{3}} \frac{\omega^{\nu}}{s_{3}} \psi \psi>
\end{aligned}
$$

where tildes over the meson symbols stand for their mean field values, and

$$
\begin{aligned}
& \Sigma_{s}=<G_{s} \sigma>+<\bar{\psi} \psi \frac{\partial G_{s}}{\partial s_{1}} \sigma-\bar{\psi} \gamma_{\lambda} \psi \frac{\partial G_{v}}{\partial s_{1}} \omega^{\lambda}> \\
& \Sigma_{\mu}=<G_{v} \omega_{\mu}>+\frac{1}{s_{2}}<\left(-\bar{\psi} \psi \frac{\partial G_{s}}{\partial s_{2}} \sigma+\bar{\psi} \gamma_{\lambda} \psi \frac{\partial G_{v}}{\partial s_{2}} \omega^{\lambda}\right) \bar{\psi} \gamma_{\mu} \psi>.
\end{aligned}
$$

These results are simplified within the assumptions (a-c) above, for the sake of completeness we consider separately each of these cases:
a) $\Sigma_{s}=G_{s} \tilde{\sigma}, \Sigma_{\mu}=G_{v} \tilde{\omega}_{\mu}+\left(d G_{v} / d n\right) \tilde{\omega}^{\nu} \Lambda_{\nu \mu}-\left(d G_{s} / d n\right) \tilde{\sigma} \Lambda_{\mu}, \tilde{\sigma}=G_{s} n_{s} / m_{s}^{2}$, $\tilde{\omega}_{\mu}=G_{v} n_{\mu} / m_{v}^{2}$, with $\Lambda_{\nu \mu}=<\bar{\psi} \gamma_{\nu} \psi \bar{\psi} \gamma_{\mu} \psi>/ n$, and $\Lambda_{\mu}=<\bar{\psi} \psi \bar{\psi} \gamma_{\mu} \psi>/ n$,
b) $\Sigma_{s}=\tilde{\sigma}\left(G_{s}+n_{s} d G_{s} / d n_{s}\right), \Sigma_{\mu}=G_{v} \tilde{\omega}_{\mu}+\left(d G_{v} / d n\right) \tilde{\omega}^{\nu} \Lambda_{\nu \mu}$,
$\tilde{\sigma}$, and $\tilde{\omega}_{\mu}$ have the same expressions as in (a),
c) $\Sigma_{s}=G_{s} \tilde{\sigma}, \Sigma_{\mu}=G_{v} \tilde{\omega}_{\mu}, m_{s}^{2} \tilde{\sigma}=G_{s} n_{s}+\tilde{\sigma} n_{s}\left(d G_{s} / d \sigma\right)$, $m_{v}^{2} \tilde{\omega}_{\mu}=G_{v} n_{\mu}+\left(d G_{v} / d \tilde{\omega}\right) n_{\nu} \tilde{\omega}^{\nu} \tilde{\omega}_{\mu} / s_{3}$,

we have used $G_{s, v}=<G_{s, v}>, n_{s}=<\bar{\psi} \psi>, n_{\mu}=<\bar{\psi} \gamma_{\mu} \psi>, n=n_{0}$ is the baryonic density, and we have taken into account the fact that $s_{2}=n$, as it is clear when it is evaluated in the reference system of rest matter. Finally, we have introduced the simplifying assumption that $G_{s, v}$ and its derivatives can be extracted from the expectation values, even when they are field functionals. Regarding to the expectation values $\Lambda_{\mu \nu}$ and $\Lambda_{\mu}$, they can be evaluated by using the Wick theorem and the propagator for the nucleons in the medium, giving rise to direct and exchange terms. In our calculations we have neglected the Fock terms, in which case we have obtained $\Lambda_{\mu \nu}=n_{\nu} n_{\mu} / n, \Lambda_{\mu}=n_{s} n_{\mu} / n$.

In previous investigations $[2,3,4]$, the procedure to define the functionals $G_{s}$ and $G_{v}$ consisted of including the nucleon self-energies as input into the equations

$$
\Sigma_{s}^{i n p}=G_{s} n_{s} / m_{s}^{2}, \quad \Sigma_{\mu}^{i n p}=G_{v} n_{\mu} / m_{v}^{2}
$$


where $\sum_{s}^{i n p}, \Sigma_{\mu}^{i n p}$ are the self-energies obtained with one boson exchange potentials in the Dirac-Brueckner-Hartree-Fock approach for symmetric nuclear matter. The Eqs. (10) coincide with Eqs. (8)-(9) if the rearrangement terms were omitted, in spite of the importance of these contributions for satisfying the thermodynamic consistency, as stressed in ref. [4]. At this point we deviate from the procedure of the DDHFT in two aspects; firstly, we adopt as input the nucleon self-energies $\Sigma^{\mathrm{QCDSR}}$ grounded on the fundamental theory of strong interactions. Secondly, we insert this input into the full expressions (8) and (9), in fact we regard from the very beginning the field dependence of $G_{s, v}$. Therefore, the vertices are the solution of a differential equation which must be solved together with the self-consistent condition for $\tilde{\sigma}$ in cases a) and b), or a pair of differential equations for $\tilde{\sigma}$ and $\tilde{\omega}$ in the case $\mathrm{c}$ ). The explicit form of these solutions are shown below, for each of the instances considered
a) $G_{s}={\frac{\Sigma_{s}}{\tilde{\sigma}}}^{\mathrm{QCDSR}}, G_{v}^{2}=2\left(\frac{m_{v}}{n}\right)^{2} \int_{0}^{n} d n^{\prime}\left[\Sigma_{v}^{\mathrm{QCDSR}}+\left(\frac{n_{s}}{m_{s}}\right)^{2} G_{s} \frac{d G_{s}}{d n}\right]$,
b) $G_{s}^{2}=2\left(\frac{m_{s}}{n_{s}}\right)^{2} \int_{0}^{n} d n^{\prime} \frac{d n_{s}}{d n} \Sigma_{s}^{\mathrm{QCDSR}}, G_{v}^{2}=2\left(\frac{m_{v}}{n}\right)^{2} \int_{0}^{n} d n^{\prime} \Sigma_{v}^{\mathrm{QCDSR}}$,
c) $\tilde{\sigma}^{2}=\frac{2}{m_{s}^{2}} \int_{0}^{n} d n^{\prime} n_{s} \frac{d \Sigma_{s}}{d n} \quad, \tilde{\omega}^{2}=\frac{2}{m_{v}^{2}} \int_{0}^{n} d n^{\prime} n^{\prime}{\frac{d \Sigma_{v}}{d n}}^{\mathrm{QCDSR}}$,

where we used that the spatial components of tetravectors become null for isotropic matter, and we considered $\Sigma_{s, v}^{\mathrm{QCDSR}}$ as being parameterized in terms of only the baryonic density $n$. The expressions for $G_{s, v}$ become singular at zero density, nevertheless they have finite limits: $G_{s}^{2} \rightarrow m_{s}^{2} d \Sigma_{s}^{\mathrm{QCDSR}}(0) / d n, G_{v}^{2} \rightarrow m_{v}^{2} d \Sigma_{v}^{\mathrm{QCDSR}}(0) / d n$, for all the cases under consideration.

Up to this point, we have constructed a hadronic effective interaction capable of reproduce the nucleon self-energies as they were evaluated in the QCD sum-rules; therefore, our findings are subjected to the same limitations in applicability.

In ref. [14], a similar purpose was attained by adopting the results of [11] and simulating the hadronic interaction by means of four fermion couplings. The vertices are taken as functions of only $s_{2}$, they were determined in the same way as in the DDHFT, namely, using Eq. (10). Nevertheless, the authors consider that the effect of chiral fluctuations is predominant in the many body aspects of nuclear matter, and the variation of the self-energies according to QCD sum-rules calculations has a stabilization effect at high densities.

In the next step we have evaluated the energy-momentum tensor $T^{\mu \nu}$ by the canonical procedure, and finally, the energy per unit volume in the MFA is obtained by taking the in-medium expectation value of $T^{00}$. This gives

$$
E_{M F A}=\int_{0}^{p_{F}} \frac{d^{3} p}{(2 \pi)^{3}} \sqrt{p^{2}+M^{* 2}}+n_{s}\left(\Sigma_{s}^{\mathrm{QCDSR}}-G_{s} \tilde{\sigma}\right)+\frac{1}{2}\left(m_{s}^{2} \tilde{\sigma}^{2}+m_{v}^{2} \tilde{\omega}^{2}\right)
$$


where we have introduced the Fermi momentum $p_{F}$, related to the baryonic density by $n=2 p_{F}^{3} /\left(3 \pi^{2}\right)$, and the effective nucleon mass $M^{*}=M-\Sigma_{s}^{\mathrm{QCDSR}}$. It must be noticed that the sign of the scalar self-energy has been changed as regards the one used in refs. $[11,12]$, in order to adopt the practices of QHD models.

Using the thermodynamical relation $P=\mu n-E_{M F A}$ we have obtained the pressure of the nuclear matter, with the chemical potential given by $\mu=E_{F}+\Sigma_{v}$ for cases a) and b), and $\mu=E_{F}+n d \Sigma_{v} / d n$ for the remaining one, being $E_{F}=\sqrt{p_{F}^{2}+M^{* 2}}$.

A feature of isospin symmetric nuclear matter is that the energy per particle has a minimum, giving rise to bound states at zero temperature. This is the least requirement that a model of nuclear matter should satisfy. A measure of this property is the binding energy $E_{B}=E_{M F A} / n-M$, which is well determined to have a minimum value $E_{B} \simeq-16 \mathrm{MeV}$ at the normal density $n_{s}=0.15 \mathrm{fm}^{-3}$. We have examined the binding energy in the three cases studied for the functional dependence of $G_{s, v}$, which are displayed in Fig. 1 in terms of the baryonic density. As we can see, none of these instances is able to fit the saturation properties of nuclear matter. Our results with the input taken from ref. [11] does not distinguish among the different parameterizations of the vertices; on the other hand, the case c) evaluated with the self-energies given by ref. [12] (case cD in the following) clearly stands out from the other ones. The non-monotonic behavior of the curve $\mathrm{cD}$ is more compatible with the phenomenology, more precisely the binding energy has a minimum $E_{B}=-4 \mathrm{MeV}$ at the density $n=0.8 n_{s}$, not far from the empirical saturation point.

It is apparent that the approaches a) and b) yield undistinguishable results for $E_{B}$. On the other hand, the case c) produces higher meson mean values, enhancing the energy density. This fact reverses the decreasing trend of $E_{B}$ obtained for the cases a) and b) in the right hand side of this figure, the net behavior is non-monotonous as shown there. On the other hand, the same effect applied to the increasing curves (a-b) on the left panel, reinforces the growth. Although, the small increment is not appreciable within the scale of this figure.

It has been stated that in order to obtain the slight binding energy and simultaneously a high nuclear spin-orbit potential, the scalar and vector components of the nucleon selfenergy must have nearly the same value, of roughly three hundred $\mathrm{MeV}$ for densities about $n_{s}$ [13]. This requisite is satisfied by both refs. [11] and [12]; however, as can be deduced from the results a) and b) it is not sufficient to determine conclusively the many body aspects of the system.

As the QCD sum-rules calculations are subjected to uncertainties of diverse origins, we have introduced two independent scaling factors $X_{s}$ and $X_{v}$ for each of the nucleon selfenergies used as inputs, and we have explored the range $\left|X_{s, v}-1\right|<0.25$. We have assumed these factors independent of the density, consequently their derivatives are scaled by the same magnitude. We have found that within this range of variation, only the case $(\mathrm{cD})$ is able to reproduce simultaneously the binding energy and the density of saturation with a slight scaling $X_{s} \simeq 1.19, X_{v} \simeq 1.12$. Aiming to a qualitative description for all the densities considered, we have introduced a uniform scaling of the self-energies $\Sigma_{s, v}^{\mathrm{QCDSR}}(n) \rightarrow X_{s, v} \sum_{s, v}^{\mathrm{QCDSR}}(n)$ within the scheme c).

As the next step, we have examined the behavior of the Landau parameters $F_{0}$ and $F_{1}$ for symmetric nuclear matter. They can be evaluated as the Legendre projections of the second derivative of $E_{M F A}$ with respect to the baryonic density, for a deduction see [15]. 
These parameters are very useful in regard to collective phenomena of the dense nuclear environment, as for instance, the giant monopolar and quadrupolar modes, or the sound velocity [16]. The nuclear compressibility can be evaluated as $K=3 p_{F}^{2}\left(1+F_{0}\right) / E_{F}$, its value at the equilibrium density is constrained experimentally to range between $230 \mathrm{MeV}$ $<K<270 \mathrm{MeV}$. Within the approach (cD) we have obtained $K=245 \mathrm{MeV}$, and related to it is the giant monopole mode energy $E_{M}=\omega_{0} / A^{1 / 3}$. In our calculations we have obtained $\omega_{0}=108 \mathrm{MeV}$, whereas, the experience yields $\omega_{0}^{\text {exp }}=80 \mathrm{MeV}$. On the other hand the scaled calculations lead to the excessively high values $K=660 \mathrm{MeV}$, and $\omega_{0}=180 \mathrm{MeV}$. Another interesting property is the excitation energy of the quadrupole state, given by the relation $E_{Q}=\omega_{2} / A^{1 / 3}$ with $\omega_{2}=p_{F} \sqrt{2} /\left(1.2 \mu \sqrt{1+F_{1} / 3}\right)$, yielding in our calculations $\omega_{2}=78 \mathrm{MeV}$, and $\omega_{2}=100 \mathrm{MeV}$ in the non-scaled and scaled cases, respectively. These values must be compared with the empirical result $\omega_{2}^{\text {exp }}=63 \mathrm{MeV}$. On the other hand, the numerical values obtained for $\omega_{0}$ and $\omega_{2}$ without scaling, can be well accommodated among the theoretical results for the isoscalar giant resonances in the non-linear models of HFT [17]. Finally, the sound velocity $v_{s}=p_{F}^{2}\left(1+F_{0}\right) /\left(3 \mu E_{F}\right)$ takes on the value $v_{s} / c=0.17$ and $v_{s} / c=0.28$ at the saturation density for the non-scaled and scaled instances, respectively. The magnitude of $v_{s}$ increases with density quickly, becoming acausal before $n \approx 2 n_{s}$ is reached. This feature puts severe limits to the range of applicability of the scheme.

In Fig. 2 we show the vertices $G_{s, v}$ for the $(\mathrm{cD})$ approach, in terms of their respective variables. They are well fitted by the expressions $G_{s}=a /\left(1+b s_{3}\right)$ and $G_{v}=A+B s_{4}$, with $a=7, b=-0.59 \mathrm{fm}^{-1}, A=7.83$, and $B=8.39 \mathrm{fm}^{-1}$.

To sum up, in the present work we have examined the possibility of matching QCD sumrules predictions and hadronic field theories. A successful combination would result in a profitable feedback, by giving foundations to hadronic models and corroborating simultaneously the QCD sum-rules methods and findings. In particular we have used results for the nucleon self-energy in a dense hadronic environment derived in two independent treatments of QCD sum-rules, with diverse degree of approximation. As a first step, we have studied different formal schemes of merging these inputs into hadronic field models and we have checked their ability to fit the basic nuclear matter phenomenology. We have introduced interaction vertices $G_{s}$ and $G_{v}$, which are functionals of the hadronic fields, and we have determined them by requiring the reproduction of the self-energies used as input. It must be emphasized that in this procedure we have included the full expression for the self-energies, having obtained a set of differential equations for the definition of these vertices, instead of algebraic ones,. We have found that only a parameterization of the vertices in terms of mesonic degrees is compatible with the basic features of dense nuclear matter. In addition we have presented a fitting in terms of rational functions, which make evident the non-linear quality of the meson-nucleon coupling. If we allow an uncertainty margin of $20 \%$ in the sum-rules calculations, this scheme is able to fit precisely the saturation properties of the isospin symmetric nuclear matter. Another physical quantities, which are related to collective nuclear phenomena, agree with the order of magnitude of the experimental values, although they are not admissible as a quantitative description. It must be stressed that no adjustable parameters are introduced, only the QCD sum-rules self-energies are required as input. The parameters $X_{s}, X_{v}$ were introduced only to study 
the versatility of the results.

We conclude that compatibility of QCD sum-rules and hadronic field models is feasible. However, a detailed qualitative description will require further improvements in QCD sum-rules calculations, in particular the high density QCD phenomenology should be properly included. Therefore, additional refinements and higher order calculations will be useful and deserve to be investigated. Improved results could be inserted into hadronic models to test another significative manifestations of the hadronic phase such as finite nuclei and the eventual deconfinement phase transition.

\section{References}

[1] B.D. Serot and J.D. Walecka, Adv. Nucl. Phys. 16 (1986) 1.;

B.D. Serot, Rep. Prog. Phys. 55 (1982) 1855.

[2] R. Brockmann and H. Toki, Phys. Rev. Lett. 68 (1992)3408.

[3] S. Haddad and M. Weigel, Phys. Rev. C 48 (1993) 2740.

[4] H. Lenske and C. Fuchs, Phys. Lett. B 345 (1995) 355.

[5] S. Typel and H.H. Wolter, Nucl. Phys. A 656 (1999) 331.

[6] G.E. Brown and M. Rho, Phys. Rev. Lett. 66 (1991) 2720.

[7] G.Q. Li, C.M. Ko, and G.E. Brown, Phys. Rev. Lett. 75 (1995) 4007; Nucl. Phys. A 606 (1996) 565;

G.Q. Li, C.M. Ko, G.E. Brown, and H. Sorge, Nucl. Phys. A 611 (1996) 539.

[8] M.A. Shifman, A.I. Vainshtein, and V.I. Zakharov, Nucl. Phys. B 147 (1979) 385.

[9] B.L. Ioffe, Nucl. Phys. B 188 (1981) 317.

[10] E.G. Drukarev and E.M. Levin, Nucl. Phys. A 511 (1990)679; Nucl. Phys. A 532 (1991) 695; Prog. Part. Nucl. Phys. 27 (1991) 77.

[11] T.D. Cohen, R.J. Furnstahl, D.K. Griegel, and X. Jin, Prog. Part. Nucl. Phys. 35 (1995) 221.

[12] E. G. Drukarev, M.G. Ryskin, and V.A. Sadovnikova, nucl-th/0406027;

E. G. Drukarev, et al, Phys. Rev. C 69 (2004) 065210.

[13] R.J. Furnstahl, and B.D. Serot, Nucl. Phys. A 673 (2000) 298.

[14] P. Finelli, N. Kaiser, D. Vretenar, and W. Weise, Nucl.phys. A 735 (2004) 449.

[15] T. Matsui, Nucl. Phys. A 370 (1981) 365;

J.C. Caillon, P. Gabinski, and J. Labarsouque, Nucl. Phys. A (2001) 623.

[16] S. Nishizaki, H. Kurasawa, and T. Suzuki, Nucl. Phys. A 462 (1987) 687.

[17] S.K. Patra, X. Vinas, M. Centelles, and M. Del Estal, Nucl. Phys. A 703 (2002) 240. 


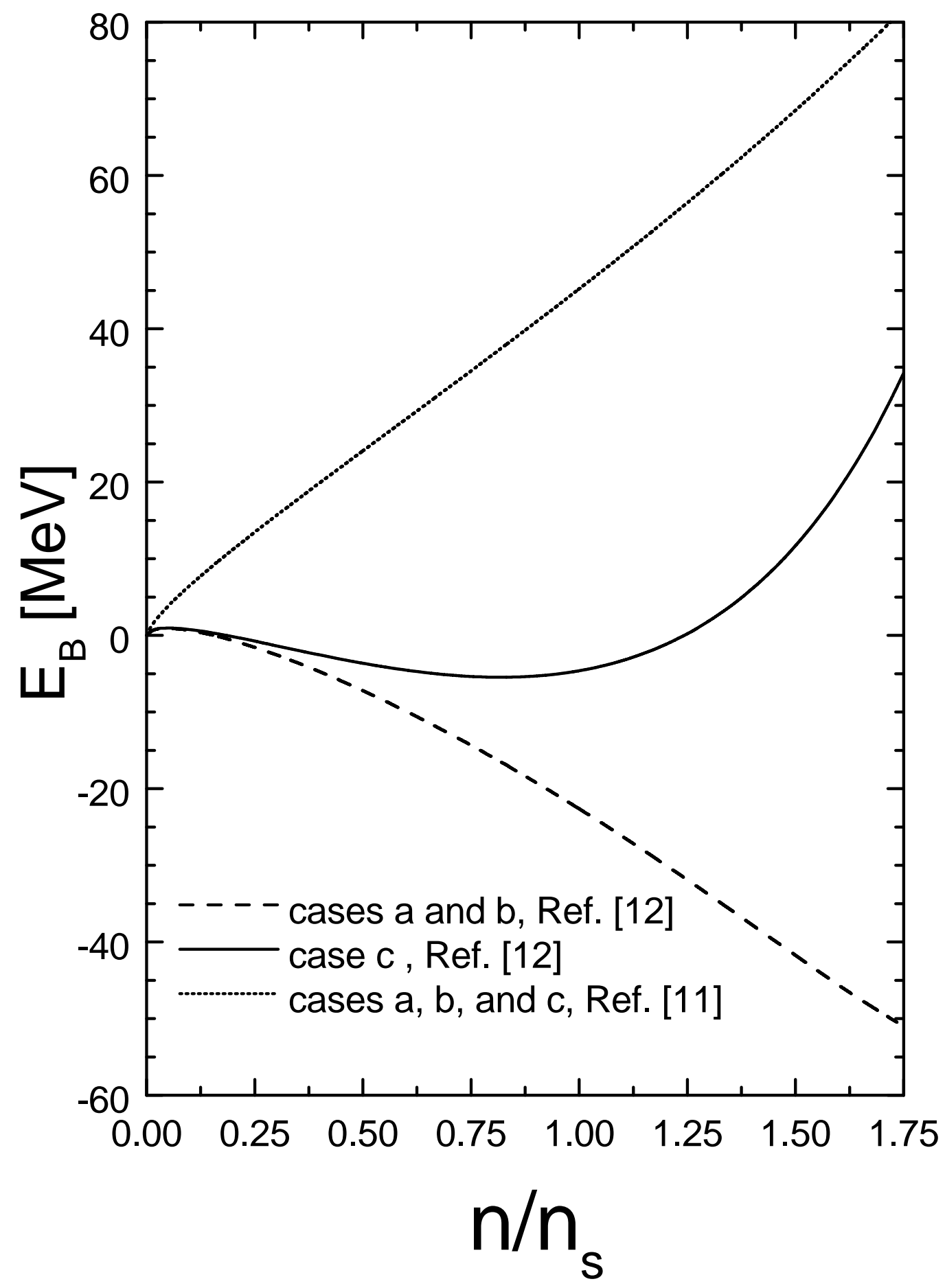

Fig. 1. The binding energy as a function of the baryonic density for each of the parameterizations of the interaction vertices, cases a-c. By using as input the self-energy given by ref. [11] all the curves coincide, in the case of including the nucleon self-energy of ref. [12] the case c) distinguishes clearly from the other ones. The line convention is indicated in the figure. 


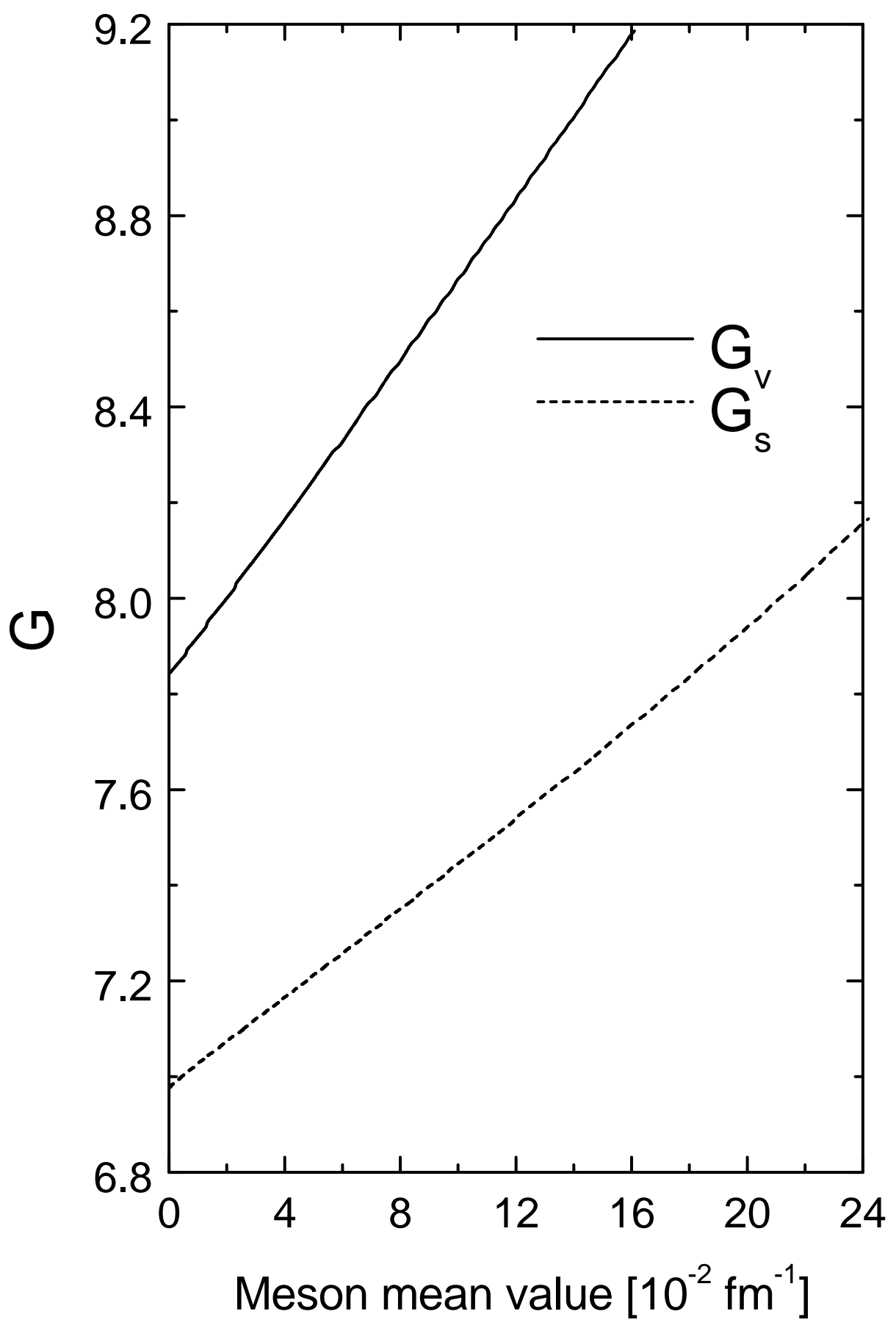

Fig. 2. The interaction vertices of the hadronic field model as functions of the meson mean field values. The model reproduces the nucleon self-energy given by ref. [12]. 